\title{
Reports
}

\section{Objective Perimetry in Sporting-Related Mild Traumatic Brain Injury}

The incidence of mild traumatic brain injury (mTBI) is increasing in the United States, where the rate of emergency department visits increased by $70 \%$ between 2007 and $2013 .^{1}$ Traumatic brain injury is defined as a traumatically induced structural injury or physiologic disruption of brain function as a result of an external force. $^{2}$ The timely diagnosis of mTBI remains challenging because of absence of serious functional impairment or loss of consciousness and is often underdiagnosed on the sporting field and in motor vehicle accidents. Concussion and mTBI are terms that have overlapping definitions, and the symptoms may be prolonged. Traditionally, acute mTBI is defined as recovery from postconcussive symptoms in less than 10 days. $^{3}$

Multifocal pupillographic objective perimetry (mfPOP) is a noncontact method, which like multifocal visual evoked potentials (mfVEPs), concurrently measures both response sensitivity and delay at many visual field locations. Both eyes are assessed in 7 minutes. We have recently added to the growing evidence, that like mfVEPs, mfPOP can inform us about both cortical and mid-brain function. ${ }^{4}$ Changes in central retinal thickness ${ }^{5}$ and visual field sensitivity ${ }^{6}$ have been reported separately in mTBI. Here we use the abilities of mfPOP to examine both sensitivity and delay at each visual field location and then correlate these measures with local Early Treatment Diabetic Retinopathy Study (ETDRS) central retinal thickness data. We also examine retinal nerve fiber layer loss.

Our prospective study contained 12 male athletes $(22.3 \pm 2.5$ years, mean \pm standard deviation) with acute mTBI, 24 male athletes with chronic mTBI (20.5 \pm 1.5 years), 10 male athletes with no history of head injury ( $22.3 \pm 2.4$ years), all recruited from local Canberra rugby clubs, and 8 age-matched controls $(22.1 \pm 1.9$ years). The mTBI subjects' concussions had removed them from the field of play, and they were separated into acute subjects, who had sustained their concussion within 36 days of testing, and a chronic group (39-667 days since concussion). They were compared with age- and sex-matched controls.

All participants underwent a series of eye examinations including visual acuity, Humphrey Matrix perimetry N 30-5 screening testing (Carl Zeiss Meditec, Dublin, CA), and OCT (Spectralis, Heidelberg Engineering, Heidelberg, Germany). The OCT measured peripapillary retinal nerve fiber layer thickness and a posterior pole scan to provide the ETDRS grids thickness data. Multifocal pupillographic objective perimetry was collected ${ }^{4}$ using a prototype of the Food and Drug Administration-cleared Konan objective FIELD Analyzer (Irvine, CA).

There was no significant difference in full retinal thickness or acuity between mTBI groups and controls and only marginally significant reductions in peripapillary retinal nerve fiber layer. The largest reduction in response amplitude (Table S1, available at www.aaojournal.org), $-2.02 \pm 0.38$ decibels $(\mathrm{dB})$, was seen in the participants with acute mTBI $(P<0.0001)$. Response amplitudes in athletes with chronic mTBI were not significantly different from controls. Interestingly, athletes with no apparent history of mTBI showed a small, but significant, mean reduction in direct response amplitude $(-1.09 \pm 0.4 \mathrm{~dB}, P=0.006)$. Response delay (time to peak) showed no significant effect in participants with acute mTBI $(12.7 \pm 6.7 \mathrm{~ms}, P=0.06)$. There was a shift toward significantly shorter delays than controls in athletes with chronic mTBI $(-13.5 \pm 6.7 \mathrm{~ms}, P=0.04)$. Uninjured athletes also demonstrated a trend to quicker time-to-peak responses $(-7.1 \pm 7.0 \mathrm{~ms}, P=0.31)$.

In athletes with acute mTBI, regional full retinal thickness showed a trend toward increased thickness (Table S2, available at www.aaojournal.org), but this did not reach significance. Regional changes in retinal nerve fiber layer thickness trended toward reduced thickness in acute mTBI relative to controls but failed to reach significance.

Average regional amplitudes in the acute mTBI group showed significantly reduced responses relative to controls in 40 of 44 regions (Fig 1A). Conversely, regional mean response delays in this group (Fig 1B) were less informative, with only 3 regions showing significantly greater delays (brightest regions). Athletes with chronic mTBI showed evidence of improvement in sensitivity with losses in less than one quarter of regions (9/44). Response delays demonstrated generalized shortening of time-topeak responses with 12 regions, with mostly central visual field regions reaching significance (Fig 1D).

We next examined correlation between response dysfunction and retinal thickness, which involved mapping the central 32 mfPOP regions onto 9 ETDRS equivalents. We found significant negative correlations between time-to-peak and ETDRS thickness in acute mTBI and significant positive correlations in chronic mTBI (Fig 1E). Thus, in agreement with full retinal thickness changes, we found that thicker retinas in chronic mTBI (Table S2) were significantly associated with significantly shorter delays (Table S1).

The results of this preliminary study suggest that response amplitudes are more informative than changes in time to peak in the first month after head injury. However, with the passage of time, the changes in amplitude lessen and, interestingly, the time-to-peak latencies shorten. These results, if confirmed in a larger group of participants, would suggest that alterations in pupil fields might serve as an objective functional biomarker in the detection of mTBI and, potentially, monitoring the changes that occur over time.

Mild TBI is often associated with asymptomatic subclinical damage. Our results identified nonconcussed athletes as having evidence of autonomic nervous system dysfunction in the absence of changes in visual acuity (Table S1). This may represent athletes with mTBI who are undiagnosed. It is also possible that these changes arose as the result of repetitive mild injury to the globe or head. 

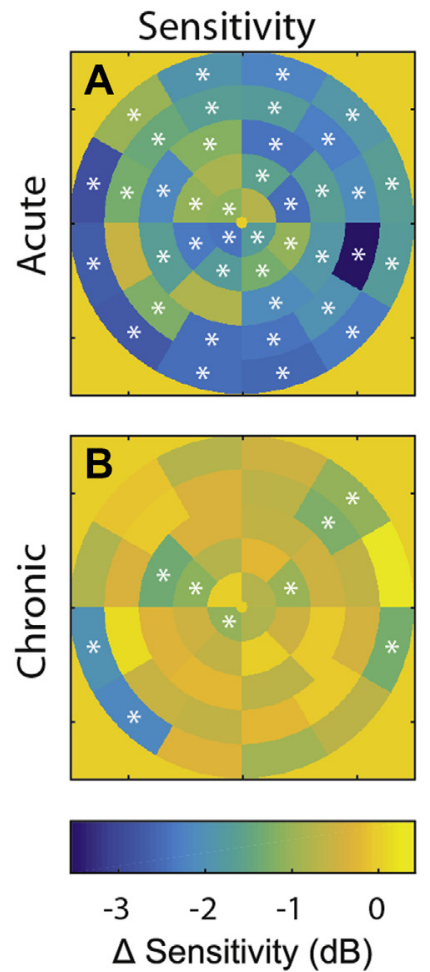
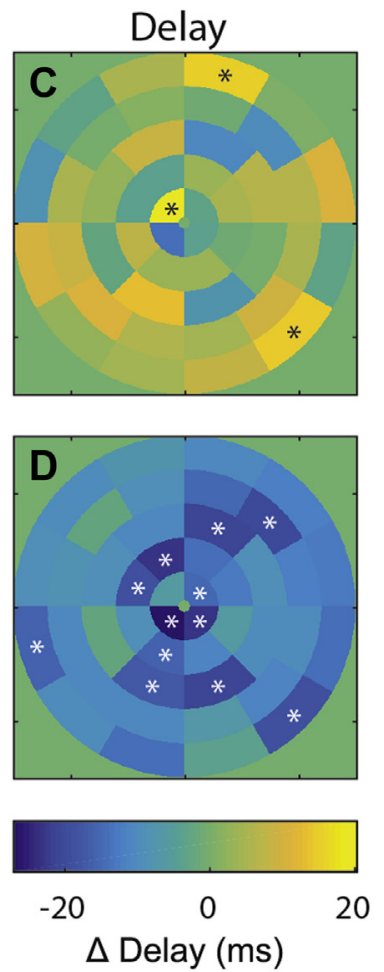

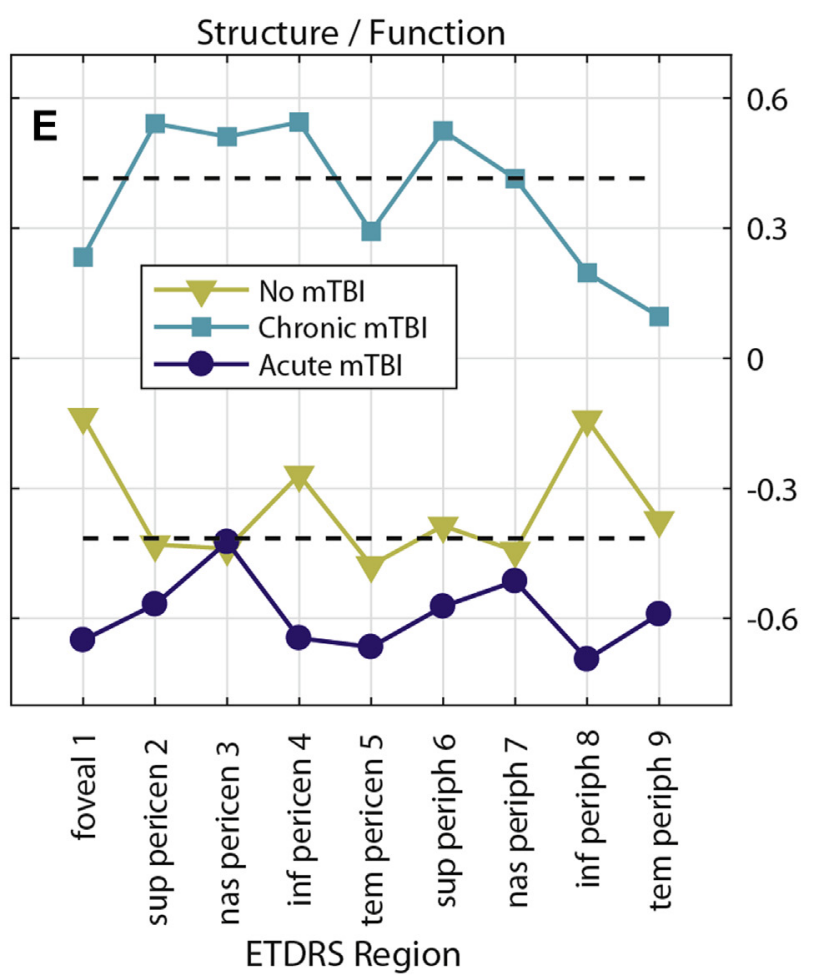

0.6

Figure 1. A-D, Regional mean pupil contraction deviations from control data. Before computing the linear models, the right eye data were flipped so that all data had the temporal field on the left. The plotted regional deviations thus represent the combined response from both eyes and all the subjects in each mild traumatic brain injury (mTBI) group relative to normal controls. Test regions with significant average deviations $(P<0.05)$ are indicated by an asterisk (*). In each of $\mathrm{A}$ to $\mathrm{D}$, the background color represents a deviation of $\mathrm{O}$. A, The mean regional deviations in sensitivity from controls for athletes with acute mTBI. A generalized depression in contraction amplitudes was found with 40 of 44 regions/eye significantly reduced compared with controls. B, Sensitivity deviations from normal for athletes with chronic mTBI. On average, response amplitudes were less affected than in athletes with acute mTBI, with only 9 regions showing significantly reduced amplitudes. C, Mean contraction delay deviations for athletes with acute mTBI relative to controls. Note that brighter regions represent longer than normal delays. D, Athletes with chronic mTBI showed predominantly shorter time-to-peak responses with the 11 darkest central regions being significantly quicker compared with normal. E, Correlations between pupillary response delay and total retinal thickness by Early Treatment Diabetic Retinopathy Study (ETDRS) region (region numbers run central to peripheral). As indicated by the legend, results are stratified for athletes with acute (dark purple), chronic (blue), and no history of mTBI (gold). The 2 dashed horizontal black lines represent levels beyond which correlations are significant $(P<0.05) . \mathrm{dB}=$ decibels.

Measurements of mfPOP are not without potential problems, and results must be interpreted with caution. For example, antidepressants such as moclobemide ${ }^{7}$ and topical antihypertensive agents including brimonidine ${ }^{8}$ may cause mydriasis of $<0.5 \mathrm{~mm}$. Decongestants, such as diphenhydramine, and stimulants, such as modafinil, have been shown to produce miosis or mydriasis, respectively, of $<0.5 \mathrm{~mm}$ and may introduce some noise in pupil diameter recordings. ${ }^{9}$ Our use of relative pupil size moderates the effects of moderate miosis or mydriasis and aging and noncircular pupils. Trauma to the iris from sports-related injury or intraocular surgery may potentially have greater effects on responses; however, preliminary reports from our laboratory on pseudophakic patients suggests only small effects. ${ }^{10} \mathrm{We}$ also use the pupil with the highest signal to noise ratio (SNR) for each test region; therefore, only 1 functional pupil is required. Systemic diseases also may affect pupil function, such as neuropathy of the pupils in diabetes. Such neuropathy mimics global depression of sensitivity, whereas we have found early- to late-stage diabetic damage is localized. ${ }^{11}$

The amount of implied retinal ganglion cell loss found in our study was variable and did not reach significance across the central $20^{\circ}$ of visual angle from the fovea in acute mTBI (Table S2). Previous pupillographic studies have focused on thalamic inputs using ganzfeld stimuli, but we have shown that the transient onset mfPOP stimuli are primarily reporting on cortical function. ${ }^{4}$ In this preliminary study, using noninvasive tests, we provide evidence of ability to track mTBI from acute to chronic stages. In particular, differences between response amplitudes and delays identified the time point of injury, and the functional changes were associated with structural changes. Change in delay and their correlation with structural changes may suggest an active compensatory mechanism. Further studies are needed to identify these hypothesized mechanisms and to explore higher cortical input to responses in mTBI. Athletes from this study will be followed up over time to monitor changes in function, and additional athletes with head injuries will be invited to participate in the study to increase the sample size. The short mfPOP test measures responses from both eyes and pupils concurrently with minimal training, and the ability to test across all age groups. Thus, mfPOP has the potential to detect and monitor mTBI. 
Faran Sabeti, PhD ${ }^{1,2}$

Corinne F. CARle, $\mathrm{PhD}^{2,3}$

RACHEL K. JAROS, BSC ${ }^{2}$

EMILIE M.F. RoHAN, BSc ${ }^{2}$

Gordon Waddington, $\mathrm{PhD}^{4}$

Christian J. Lueck, MD, PhD ${ }^{3,5}$

DAVID Hughes, MBBS ${ }^{6}$

Ted Maddess, $\mathrm{PHD}^{2}$

${ }^{1}$ Department of Optometry, University of Canberra, Canberra, ACT, Australia; ${ }^{2}$ The John Curtin School of Medical Research, Australian National University, Canberra, ACT, Australia; ${ }^{3}$ Australian National University Medical School, Australian National University, Canberra, ACT, Australia; ${ }^{4}$ Research Institute for Sport and Exercise, University of Canberra, Canberra, ACT, Australia; ${ }^{5}$ Department of Neurology, The Canberra Hospital, Canberra, Australia; ${ }^{6}$ Department of Sports Medicine, Australian Institute of Sport, Canberra, Australia

Financial Disclosure(s): The author(s) have made the following disclosure(s): C.F.C. and T.M.: Patents assigned to Konan Medical USA LLC.

Supported by National Health and Medical Research Council Project Grant APP1063458 and Rebecca Cooper Medical Foundation Grant PG2018040.

HUMAN SUBJECTS: Human subjects were included in this study. The human ethics committees by the Australian National University Human Experimentation Ethics Committee (protocol 2017/194) and the Australian Institute of Sport protocol 2015-1202 approved the study. All research adhered to the tenets of the Declaration of Helsinki. All participants provided informed consent.

No animal subjects were used in this study.

Author Contributions:

Conception and design: Waddinton, Maddess

Analysis and interpretation: Sabeti, Carle, Jaros, Lueck, Maddess

Data collection: Sabeti, Jaros, Rohan, Lueck

Obtained funding: N/A

Overall responsibility: Sabeti, Waddington, Lueck, Hughes, Maddess

Correspondence:

Faran Sabeti, PhD, Department of Optometry, Faculty of Health, University of Canberra, Bruce, ACT 2617. E-mail: faran.sabeti@ canberra.edu.au.

\section{References}

1. Taylor CA, Bell JM, Breiding MJ, Xu L. Traumatic brain injury-related emergency department visits, hospitalizations, and deaths - United States, 2007 and 2013. MMWR Surveill Summ. 2017:66:1-16.

2. Management of Concussion/mTBI Working Group. VA/DoD Clinical Practice Guideline for Management of Concussion/ Mild Traumatic Brain Injury. J Rehabil Res Dev. 2009;46: CP1-CP68.

3. Choe MC, Giza CC. Diagnosis and management of acute concussion. Semin Neurol. 2015;35:29-41.

4. Sabeti F, James AC, Carle CF, et al. Comparing multifocal pupillographic objective perimetry (mfPOP) and multifocal visual evoked potentials (mfVEP) in retinal diseases. Sci Rep. 2017:7:45847.
5. Bixenmann B, Bigsby K, Hasselfeld KA, et al. Retinal and balance changes based on concussion history: a study of division 1 football players. Int $J$ Phys Med Rehabil. 2014;2:1-6.

6. Walsh DV, Capo-Aponte JE, Jorgensen-Wagers K, et al. Visual field dysfunctions in warfighters during different stages following blast and nonblast mTBI. Mil Med. 2015;180: $178-185$.

7. Bitsios P, Langley RW, Tavernor S, et al. Comparison of the effects of moclobemide and selegiline on tyramineevoked mydriasis in man. Br $J$ Clin Pharmacol. 1998;45:551-558.

8. Kesler A, Shemesh G, Rothkoff L, Lazar M. Effect of brimonidine tartrate $0.2 \%$ ophthalmic solution on pupil size. J Cataract Refract Surg. 2004;30:1707-1710.

9. Hou RH, Langley RW, Szabadi E, Bradshaw CM. Comparison of diphenhydramine and modafinil on arousal and autonomic functions in healthy volunteers. J Psychopharmacol. 2007;21: $567-578$.

10. Kolic M, Maddess T, Essex RW, James AC. Effect of intraocular lens implants on diagnostic performance of multifocal objective perimetry in glaucoma. ARVO-IOVS. 2010:51 E-Abstract 5513.

11. Sabeti F, Nolan CJ, James AC, et al. Multifocal pupillography identifies changes in visual sensitivity according to severity of diabetic retinopathy in type 2 diabetes. Invest Ophthalmol Vis Sci. 2015;56:4504-4513.

\section{Impact of Vision Loss on Health-Related Quality of Life in Trinidad and Tobago}

Estimating quality-adjusted life years (QALYs) lost to vision impairment (VI) and the cost effectiveness of alternative interventions requires reliable measurement of health-related quality of life. Whether VI impacts health-related quality of life is uncertain, ${ }^{1}$ but recent population-based surveys in Singapore and South Korea established an independent association between severe distance VI and utility loss. Here we report novel independent association between less severe categories of distance and near VI (NVI) in adult participants 40 years of age and older in the National Eye Survey of Trinidad and Tobago.

The 2013 through 2014 National Eye Survey of Trinidad and Tobago is the most comprehensive Caribbean eye survey undertaken in recent decades. Details are provided elsewhere. ${ }^{2}$ Briefly, we sampled 9913 eligible people 5 years of age and older, including 4263 who were 40 years of age or older, residing in 3556 households (95.9\% coverage) in 120 clusters, using multistage, random cluster sampling with probability-proportional-to-size methods. We measured presenting distance and near visual acuity outside households using logarithm of the minimum angle of resolution (logMAR) charts at $3 \mathrm{~m}$ and $40 \mathrm{~cm}$, respectively, and standard measurement protocols (stage 1$)$. We defined mild VI (0.32-0.48 $\log$ MAR), moderate VI $(0.50-1.00 \quad \log M A R)$, severe VI (1.02-1.30 $\log$ MAR), and blindness $(\geq 1.32 \log$ MAR $)$ in the betterseeing eye, and binocular NVI $(0.32-1.30 \log$ MAR at $40 \mathrm{~cm}$ with $\leq 0.30 \log$ MAR at $3 \mathrm{~m}$ ). Avoidable VI included uncorrected refractive error, cataract, diabetic retinopathy, and glaucoma.

All participants 40 years of age or older were invited for clinicbased assessment (stage 2), including EQ-5D-5L. ${ }^{3}$ EQ-5D-5L 